\title{
Risikoorientierte Analyse und Handhabung von Anforderungsänderungen
}

Iris Gräßler ${ }^{1}$, Christian Oleff ${ }^{1}$

${ }^{1}$ Research Group for Product Creation, Heinz Nixdorf Institute, Paderborn University, Germany

\section{Abstract}

Requirement changes are a major cause for project failure. A systematic approach to manage those changes from the very beginning should be an integral part of each development project. Although this is accepted in both science and industry, there is no adequate approach to tackle the issue, especially in the context of interdisciplinary systems. In this paper, a secondary analysis is done to identify all information that is necessary to manage those changes efficiently. The demanded information is pictured in a reference model and then mapped with the capabilities of existing approaches. Based on this, research gaps are identified and used to guide future research efforts.

Keywords: Requirements Engineering, Requirements Change Management, Product Development, Reference Model

\section{Motivation}

Eine wesentliche Herausforderung bei der Entwicklung komplexer interdisziplinärer Systeme, an der eine Vielzahl an Entwicklungsvorhaben scheitern [1], ist der effiziente Umgang mit Anforderungsänderungen. Anforderungsänderun- 
gen sind auf das dynamische Projektumfeld und den fortschreitenden Erkenntnisgewinn in der Entwicklung zurückzuführen. Sie können somit projektexterne als auch -interne Ursachen haben [3]. Die Änderungsauswirkungen sind insbesondere durch die Verknüpfungen zwischen den Anforderungen schwerwiegend. Falls Abhängigkeiten bei der Implementierung unberücksichtigt bleiben, kann dies zu unwirtschaftlichen Entscheidungen oder inkonsistenten Entwicklungszielen führen. Eine Gefährdung des Projekterfolgs kann nur durch vorausschauende und durchgängige Berücksichtigung von Anforderungsänderungen vermieden werden.

\section{Problemstellung und Forschungsziel}

Zur ganzheitlichen Handhabung von Anforderungsänderungen in interdisziplinären Entwicklungsprojekten ist es erforderlich, systematisch alle Informationen hinsichtlich Änderungswahrscheinlichkeit und -auswirkungen zu sammeln. Für interdisziplinäre Systeme ist dies besonders wichtig, da disziplinübergreifende Abhängigkeiten bestehen, die nur bei einem ganzheitlichen Vorgehen ausreichend berücksichtigt werden [4]. Geeignete Präventions- und Reaktionsmaßnahmen müssen vorgehalten werden. Eine entsprechende Wissensbasis mit darauf abgestimmten Maßnahmen gibt es bisher nicht. In der Softwaretechnik und der technischen Produktentwicklung sind einzelne Methoden beschrieben, die einen oder mehrere Aspekte des Problems adressieren. Ein ganzheitlicher Ansatz, der Anforderungsabhängigkeiten ausreichend berücksichtigt oder präventive Maßnahmen unterstützt, fehlt. Als ganzheitlich wird die Unterstützung aller Entscheidungen prospektiv, begleitend und retrospektiv zum Auftreten eines Anforderungsänderungsbedarfs definiert. Aus dieser Lücke folgt der Forschungsbedarf, dass eine strukturierte Untersuchung erfolgt, welche Informationen für die Anforderungsänderungsanalyse und -handhabung erforderlich sind und welche Ansätze zu deren Erhebung existieren. Das Forschungsziel ist daher die Beantwortung folgender Fragen:

1. Welche Informationen sind für die ganzheitliche Handhabung von Anforderungsänderungen in der interdisziplinären Produktentwicklung erforderlich?

2. Welche in der Literatur beschrieben Ansätze zielen explizit auf die Erhebung von Informationen für die ganzheitliche Handhabung von Anforderungsänderungen $a b$ ?

\section{Vorgehensweise}

In diesem Beitrag werden Forschungsergebnisse vorgestellt, die sich in ein übergeordnetes Forschungsvorhaben eingliedern. Dies ist entsprechend Typ 5 der DRM nach Blessing und Chakrabarti [5] strukturiert und zielt in den 
ersten beiden Stufen (Research Clarification und Descriptive Study I) auf die Determinierung von Forschungsbedarf und -fokus und die Erarbeitung eines tiefgreifenden Verständnisses des Forschungsfelds ab. Zwei zentrale Elemente dafür sind die Ausarbeitung eines initialen Referenzmodells der Ausgangssituation (Reference Model) und in diesem Fall die Durchführung einer umfangreichen Sekundäranalyse über alle relevanten Forschungsfelder hinweg. Zunächst wird die Sekundäranalyse (Kapitel 4) durchgeführt. Der Fokus lag auf Konferenz- und Journalbeiträge (peer-reviewed) sowie Literaturstudien, die in einer der folgenden Literaturdatenbanken erfasst sind: IEEE Explore, SpringerLink, Science Direct, Google Scholar, Web of Science und Design Society. Ziel war die strukturierte Erfassung relevanter und qualitativ hochwertiger Forschungsbeiträge sowie die Ausarbeitung eines Referenzmodells im Sinne der DRM. Mit Hilfe des Referenzmodells (Kapitel 5) kann der derzeitige Stand der Wissenschaft hinsichtlich aller wesentlichen Einflussfaktoren auf Anforderungsänderungen sowie deren Effekte auf den Projekterfolg visualisiert werden [5]. Anschließend wird aus dem Referenzmodell abgeleitet, über welche Einflussfaktoren Wissen aufgebaut werden sollte, damit eine risikoorientierte Handhabung von Anforderungsänderungen erfolgen kann. Zuletzt werden zur Beantwortung der zweiten Forschungsfrage diese Informationsbedarfe mit den aus der Sekundäranalyse bekannten Ansätzen abgeglichen (Kapitel 6).

\section{Stand der Technik}

In diesem Kapitel werden die methodischen Randbedingungen für die Handhabung von Anforderungsänderungen erläutert. Anschließend wird auf den Prozess zur Vorbereitung, Entscheidung und Umsetzung von Änderungen eingegangen und relevante Forschungsfelder aufgezeigt, aus denen existierende Ansätze auf den Kontext von interdisziplinären Systemen übertragen werden können.

\subsection{Anforderungsänderungen}

Anforderungsänderungen sind unvermeidbar in einem Entwicklungsprojekt [2]. Begründet liegt dies im Neuheitsgrad und der damit verbundenen Unsicherheit. Anforderungen werden beispielsweise durch neue Erkenntnisse im Entwicklungsverlauf oder veränderte Rahmenbedingungen ergänzt, angepasst oder entfernt. Dies geschieht kontinuierlich und führt zu einem kontinuierlichen Wandel der Anforderungsliste im Projektverlauf (vgl. Bild 1). Anforderungsänderungen sind nicht auf ein einzelnes Element begrenzt, sondern ziehen in vielen Fällen eine Änderungskaskade nach sich (Änderungspropagation). Grund dafür ist, dass die Anforderungen durch unterschiedliche Abhängigkeiten mit- 
einander in Wechselwirkung stehen. Ob der Propagationseffekt aktiv gehandhabt werden muss und vernachlässigbar ist, muss daher fallspezifisch bewertet werden [4].

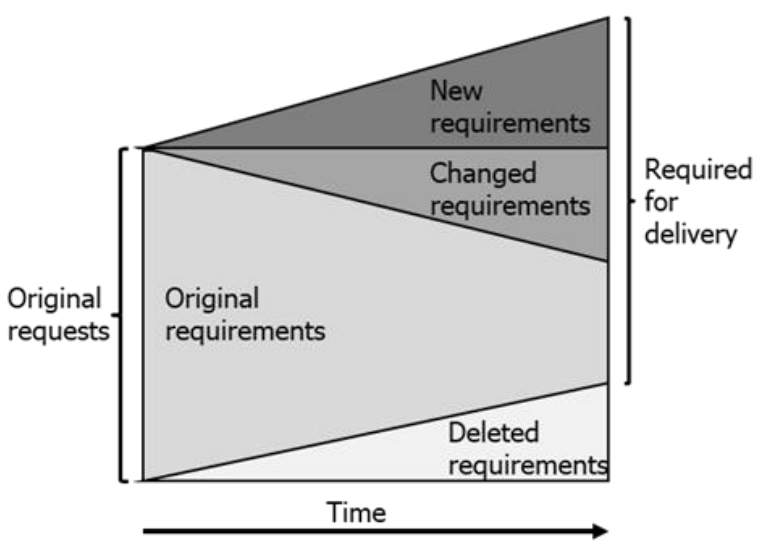

Bild 1: Anforderungsänderungen im Laufe der Produktentwicklung [6]

Die Ursachen für eine Anforderungsänderung sind vielfältig. Zunächst ist zwischen einem initialen Änderungsimpuls und einer Änderungspropagation zu unterscheiden. Während Propagationseffekte zwischen Anforderungen erfolgen, kann der initiale Änderungsimpuls fünf Ursprungskategorien zugeordnet werden: Externes Marktumfeld, Kundenorganisation, Projektvision, Anforderungsspezifikation und Lösung [7]. Innerhalb der Kategorien wird des Weiteren unterschieden, ob eine Änderung durch Unsicherheit bzw. unvollständiges Wissen hervorgerufen wird oder durch einen expliziten Auslöser („trigger"). Die Kategorie externes Marktumfeld umfasst bei dieser Kategorisierung sowohl Veränderungen von Regularien als auch von Kundengruppen. Ein Wandel der Vorgaben und Ziele eines einzelnen Kunden hingegen wäre der Kategorie Kundenorganisation zuzuordnen. Gemeinsam repräsentieren diese beiden Kategorien Änderungsimpulse, die von außerhalb der Unternehmung auf das Entwicklungsprojekt einwirken. Im Gegensatz dazu stehen die verbleibenden drei Kategorien für Änderungsbedarfe, die auf das Entwicklungsprojekt selbst zurückzuführen sind. Entgegen der Wahrnehmung von Entwicklern, überwiegen in der Praxis die Änderungsbedarfe aus dem Unternehmen selbst. Beispielsweise nehmen nachträgliche Anforderungsänderungen, die auf unzureichendes Requirements Engineering zurückzuführen sind, einen Anteil von über $50 \%$ aller Änderungen ein. Dazu zählen unvollständig erfasste Anforderungen, doppeldeutige Formulierungen, fehlerhafte Dekomposition oder Redundanzen. Veränderungen der Kundenanforderungen hingegen repräsentieren über den gesamten Projektverlauf hinweg nur etwa 15\% aller Änderungen. [3] 


\section{2 Änderungsmanagement}

Die Anzahl und Auswirkungen selbst induzierter Änderungen können durch einen systematischen Änderungsprozess verringert werden. In der Literatur werden unterschiedliche Änderungsprozesse beschrieben $[8,9]$. Zeitlich sind dort die Phasen vor dem Änderungsbedarf („pre change"), die Änderungsentscheidung („,in change") und die Änderungsimplementierung („post change") zu unterscheiden [10]. Etablierte Änderungsprozesse fokussieren sich vorwiegend auf die Implementierungsentscheidung (Akzeptanz/Ablehnung und Implementierungsgrad) sowie die Umsetzungsunterstützung $[8,9,11]$. Die Auswirkungsanalyse ist dabei Kernaktivität aller Referenzprozesse. Ziele des Änderungsmanagements sind laut Wickel die Vermeidung, Vorverlagerung, Effizienz, Effektivität und das Lernen [12]. Vermeidung soll unnötigen Änderungen vorbeugen und dadurch die Gesamtzahl der Änderungen minimieren. Vorverlagerung zielt durch eine frühe Handhabung auf geringere Auswirkungen der Änderung ab. Beide Zielsetzungen sind präventive Steuerungsmaßnahmen, die vor einem expliziten Änderungsbedarf wirken. Effektivität ist die Akzeptanzquote bei einem eingetretenen Änderungsbedarf ausgerichtet und gilt der Ablehnung von unwirtschaftlichen Änderungen (Nutzen < Aufwand). Nachdem eine Änderung akzeptiert wurde, kann das Ziel Effizienz verfolgt werden. Der Fokus liegt hierbei auf einer aufwandsarmen und schnellen Implementierung. Das letzte Ziel Lernen, beabsichtigt eine kontinuierliche Verbesserung hinsichtlich aller zuvor genannten Ziele. In der Praxis weicht das Vorgehen vom theoretischen Prozess ab. Zum einen werden häufig nur die Phasen Änderungsentscheidung und -implementierung betrachtet. Zum anderen stehen die Ziele Effektivität und Effizienz im Vordergrund [13]. Das führt dazu, dass Änderungen erst aktiv gehandhabt werden, sobald der Änderungsbedarf vorliegt und das nun erforderliche Vorgehen (Entscheidung und Durchführung) einzig auf den Erfahrungswerten der Beteiligten beruht $[4,13]$.

\subsection{Handhabung von Anforderungsänderungen}

Die Sekundäranalyse hat gezeigt, dass bisher kaum Ansätze vorliegen, die sich explizit mit Anforderungsänderungen für interdisziplinäre Systeme befassen. Dementsprechend bleiben die Informationsbedarfe und Einflussfaktoren auf Anforderungsänderungen weitestgehend unklar. Lediglich die Bedeutung von Anforderungsabhängigkeiten wird betont $[12,14]$. Wenn interdisziplinäre Systeme im Vordergrund stehen, fokussieren sich Veröffentlichungen auf einzelne Aspekte wie beispielsweise Änderungsursachen [3] oder Änderungsvorhersagen $[15,16]$. Statt eines Fokus auf interdisziplinäre Systeme und deren Anforderungen, ist die überwiegende Anzahl der Veröffentlichungen den beiden Forschungsfelder Engineering Change Management (ECM) und Requirement 
Change Management (RCM) zuzuordnen. ECM ist ein sehr weitläufiges Forschungsfeld, in dem die Änderungen von Komponenten im Vordergrund stehen. Eine umfangreiche Literaturstudie von Hamraz et al. aus dem Jahr 2013 [10] untergliedert das Themenfeld in die Phasen vor, während und nach einer Änderung. Je nach Phase steht entweder eine Vermeidung [12], Handhabung [17] oder Auswirkungsanalyse [18] im Vordergrund. Wickel baut darauf auf und bezieht mit dem Ziel einer (Teil-) Automatisierbarkeit die Datenerhebung mit ein [12]. Dafür wird zwischen Interview- oder Datenbank-basierten Ansätzen unterschieden. Hintergrund ist, dass in der industriellen Anwendung nur durch eine (teil-) automatisierte Datenerhebung und -verarbeitung eine umfangreiche Datenbasis erstellt und ausgewertet werden kann. Obwohl einige ECM-Ansätze statt Komponenten auch andere Aspekte wie beispielsweise Prozesse oder Personen adressieren, werden Anforderungen nur vereinzelt unbeachtet [19]. Bedeutsam ist dies, da durch die Betrachtung von Anforderungen zusätzlich die Phase vor einem Änderungsbedarf und dementsprechend die beiden Änderungsmanagement-Ziele Vermeidung und Vorverlagerung adressiert werden können. Dennoch bergen zahlreiche ECM-Ansätze das Potenzial in Gänze oder in Teilen auf Anforderungsänderungen übertragen zu werden. Dies gilt insbesondere für die Auswirkungsanalyse und Änderungsimplementierung. Auch Ansätze die historische Daten auswerten und einen Lerneffekt begünstigen [20, 21] sind vielversprechend für eine Übertragung auf Anforderungen interdisziplinärer Systeme. Ansätze des RCM sind ebenfalls relevant. Veröffentlichungen sind überwiegend aus der Softwaretechnik und zielen daher auf Softwareanforderungen ab. Dementsprechend lassen sich beispielsweise physikalische Abhängigkeiten nicht mit den Ansätzen verarbeiten. Zudem stützen sich viele Ansätze auf Modelle oder Daten, die in einem Softwareentwicklungsprojekt üblicherweise vorhanden sind, nicht aber für alle Elemente eines interdisziplinären Systems (z.B. UML Diagramm). Dies lässt sich aus einer Literaturstudie von Jayatilleke und Kai aus dem Jahr 2018 [11] schlussfolgern. Die Sekundäranalyse zeigt jedoch auch, dass aus der Softwaredisziplin vielversprechende Ansätze beispielsweise zur Automatisierung des Änderungsprozesses abgeleitet werden können [14]. Für die Softwareentwicklung ist die Bedeutung von Anforderungsänderungen schon seit geraumer Zeit anerkannt, dementsprechend sind einige Ansätze sehr fortschrittlich hinsichtlich praktischer Anwendbarkeit, Auswirkungsanalyse und Aufwandsminimierung [22].

\section{Informationsbedarfe für die effiziente Handhabung von Anforde- rungsänderungen}

Auf Grundlage der Sekundäranalyse werden im folgenden Informationsbedarfe abgeleitet, durch die eine ganzheitliche Handhabung von Anforderungsänderungen in der interdisziplinären Produktentwicklung ermöglicht wird. Als 
übergeordnetes Managementinstrument für Änderungen in einem Projekt, wird zunächst der Änderungsprozess betrachtet. Anschließend werden Informationsbedarfe aufgezeigt, die für einzelne Anforderungen gelten und in ihrer Gesamtheit die Wissensgrundlage für den Änderungsprozess darstellen.

Für eine effiziente Handhabung von Anforderungsänderungen ist auf oberster Ebene Wissen über die Auswirkungen der einzelnen Anforderungsänderung auf den Projekterfolg in Kombination mit der Anzahl an durchgeführten Änderungen erforderlich. Anzahl und Auswirkungen von Änderungen sind die Gütekriterien für den Änderungsprozess [8]. Der Prozess selbst zielt, wie beschrieben, durch Präventivmaßnahmen („Vermeidung" und "Vorverlagerung"), eine effektive Implementierungsentscheidung („Effektivität") und Effiziente Implementierung („Effizienz") auf die Minimierung dieser beiden Faktoren ab. Das Ziel "Lernen" ist von großer Bedeutung in der Praxis, aber nur ein indirekter Einflussfaktor und daher nicht berücksichtigt. Das Ziel Prävention wurde als umfassendere Kategorie definiert, da neben den in Kapitel 4.2 erörterten Änderungszielen - der Vermeidung und der Vorverlagerung - auch andere Risikosteuerungsmaßnahmen möglich sind. Beispiele dafür sind eine vertragliche Risikoüberwälzung oder konstruktive Beeinflussung der Wechselwirkungen [13]. Präventionsmaßnahmen können sowohl die Anzahl der Änderungen als auch deren Auswirkungen reduzieren [12]. Dies unterstreicht die herausragende Bedeutung von Präventionsmaßnahmen. Die Effektivität der Implementierungsentscheidung hingegen zielt ausschließlich auf die Ablehnung unwirtschaftlicher Änderungsanfragen ab und beeinflusst nicht die Änderungsauswirkungen. Dem entgegengesetzt wird die effiziente Implementierung. Hierbei liegt das Augenmerk einzig auf der Minimierung von Änderungsauswirkungen.Insbesondere Entscheidungen zum Einsatz von Präventionsmaßnahmen erfordern ein profundes Wissen über das Änderungsrisiko. Zur Beurteilung des Risikos werden die zwei Beurteilungskriterien Auswirkung und Eintrittswahrscheinlichkeit einer Anforderungsänderung verwendet [4, 23]. Da ein Risiko nur solange besteht, bis das zugrundeliegende Ereignis (expliziter Änderungsbedarf) eintritt, ist dieses Wissen lediglich bis zum Zeitpunkt der Implementierungsentscheidung von Bedeutung. Die Änderungswahrscheinlichkeit lässt sich zunächst auf zwei wesentliche Einflussbereiche zurückführen. Einerseits können Änderungen eine Vielzahl unterschiedlicher Ursachen haben (vgl. Kapitel 4.1). Diese Änderungsquellen sind aber nur relevant, wenn ein initialer Änderungsimpuls eingebracht wird. Andererseits sind auch Propagationseffekte zu berücksichtigen, die zwischen den Anforderungen wirken [24]. Eine Fallstudie im Kontext eines Softwareentwicklungsprojekts zeigte sogar, dass nur die Anzahl der Abhängigkeiten in einem Anforderungsnetz ein geeigneter Indikator für die Änderungswahrscheinlichkeit einer Anforderung ist [7]. Für eine fun- 
dierte Wahrscheinlichkeitsabschätzung ist daher sowohl Wissen über die initialen als auch über die sekundären Änderungsursachen erforderlich. Zur Beurteilung der Risikodimension Änderungsauswirkung sind die Kriterien Kosten und Zeit entscheidend. Eine Detaillierung dieser Faktoren ist im Rahmen dieses Referenzmodells nicht sinnvoll, da sie je nach System und Rahmenbedingungen sehr vielfältig definiert werden. Die Qualitätsdimension kann in diesem Fall vernachlässigt werden, da Anforderungen zu jedem Zeitpunkt die Kundenbedürfnisse wiederspiegeln sollten und somit die bestmögliche Qualität abbilden. Das Wissen über die Änderungsauswirkungen basiert auf den individuellen Effekten auf die Anforderung selbst und auf den Abhängigkeiten im Anforderungsnetzwerk. Die Abhängigkeiten sind für interdisziplinäre Systeme von besonderer Bedeutung, da es auch fachbereichs- und disziplinübergreifende Wechselwirkungen und somit Propagationseffekte gibt. Vor allem diese Abhängigkeiten entziehen sich bei einer rein erfahrungsbasierten Beurteilung der fachlichen Expertise des Entwicklers [4]. Eine systematische Erfassung unter Einbeziehung unterschiedlicher Fachexperten ist daher erforderlich [13]. Im Gegensatz zum Änderungsrisiko ist das Wissen über die Änderungsauswirkungen aber auch nach Eintreten eines Änderungsbedarfs erforderlich und ein zentraler Faktor für eine effektive Implementierungsentscheidung.Abschließend ist der Zusammenhang zwischen dem Wissen über Anforderungsabhängigkeiten und der Effizienz einer Änderungsimplementierung zu berücksichtigen. Durch die Kenntnis der Abhängigkeiten können Propagationseffekte auf Anforderungs- und dadurch indirekt auf Komponentenebene frühzeitig berücksichtigt und konsistent umgesetzt werden. So sind weniger Fehlerkorrekturen in späten Entwicklungsphasen erforderlich, die beispielsweise durch Inkonsistenzen im Anforderungsset hervorgerufen werden. Die erläuterten Informationsbedarfe und deren Zusammenhänge werden in Bild 2 in Form eines Referenzmodells dargestellt.

Es lässt sich schlussfolgern, dass es drei grundlegende Informationsbedarfe gibt, aus denen sich das erforderliche Wissen zur effizienten Handhabung von Anforderungsänderungen generieren lässt. Dies sind die Volatilität der Einflussfaktoren, die Anforderungsabhängigkeiten und die individuellen Auswirkungen einer Änderung auf eine Anforderung. Die Erfassung und Aktualisierung der oben benannten Informationen sollte kontinuierlich erfolgen, zumindest aber an Meilensteinen, da dort ein Großteil der Änderungsbedarfe induziert oder identifiziert wird [25]. Dies ist in zweierlei Hinsicht wichtig. Zum einen können nur so die Weiterentwicklung der Anforderungsliste erfasst und auf neue Risiken untersucht werden. Zum anderen ist eine beständige Dokumentation wichtig, um die Experteneinschätzungen und Analyseergebnisse mit den tatsächlichen Änderungen und Auswirkungen abzugleichen. So kann das im Unternehmen vorhandene Wissen gesichert und Lernpotenziale genutzt werden. 


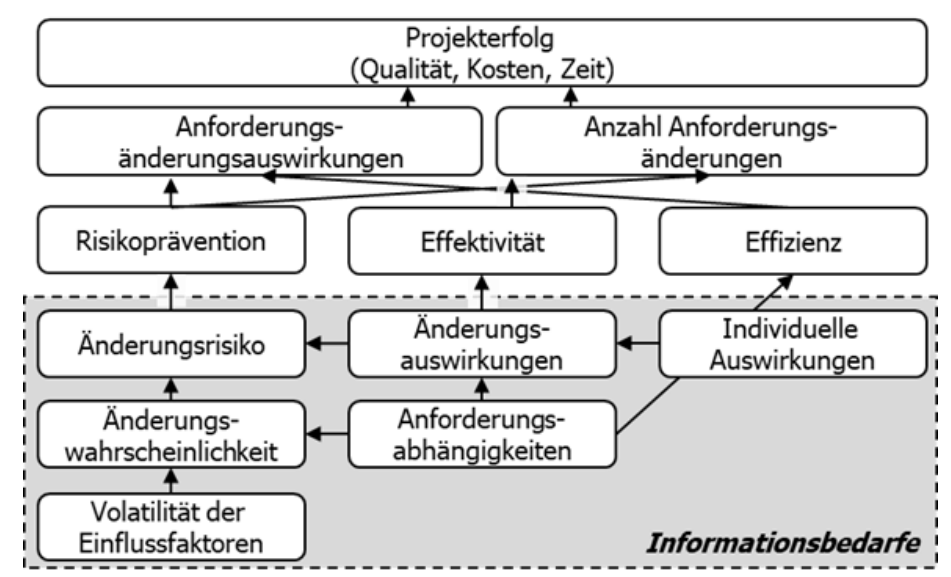

Bild 2: Referenzmodell für die Informationsbedarfe zur risikoorientierten Analyse und Handhabung von Anforderungsänderungen

\section{Forschungsbedarfe}

Auf Basis der Informationsbedarfe und der Resultate der Sekundäranalyse wurden geeignete Ansätze zur die Befriedigung der Informationsbedarfe identifiziert. Dabei wurden folgende Kriterien zugrunde gelegt:

Ermittelte Informationen: Volatilität der Einflussfaktoren; Anforderungsabhängigkeiten (direkte / indirekte und Konflikt / Synergie); Individuelle Auswirkungen (Kosten und Zeit)

Informationsgüte (Subjektivität der Daten, Vollständigkeit, Integration von historischen Daten/Lerneffekte)

Praktische Anwendbarkeit (Datenerhebungsaufwand, Skalierbarkeit, Integrierbarkeit in Unternehmensprozesse und -software)

In Tabelle 1 werden die wichtigsten Ansätze aufgeführt und der Erfüllungsgrad der einzelnen Kriterien qualitativ dargestellt. Die Auswertung zeigt unterschiedliche Forschungsbedarfe auf. Zum einen werden Einflussfaktoren kaum berücksichtigt. Dies ist wahrscheinlich darauf zurückzuführen, dass sie stark von den Spezifika des Projekts abhängen. Dennoch ist deren Berücksichtigung wichtig, da ohne die Einbeziehung von Änderungsursachen keine verlässliche Einschätzung der Änderungswahrscheinlichkeit erfolgen kann. Sowohl die Anforderungsabhängigkeiten als auch die Auswirkungen werden vielfach behan- 
delt. Hier besteht jedoch Forschungsbedarf hinsichtlich Besonderheiten interdisziplinärer Systeme. Da die Ansätze überwiegend auf eine Disziplin ausgerichtet sind, wird die Datenerhebung auf die dort verfügbaren Daten limitiert und die fachübergreifende Kommunikation zu ganzheitlichen Beurteilung von Propagationseffekten unzureichend gefördert. Interdisziplinären Systemen liegen umfangreiche Anforderungssets zugrunde, daher spielt auch die Automatisierbarkeit eine wichtige Rolle. Während zumindest eine Teilautomatisierung für Softwareanforderungen gut realisierbar ist, haben Komponentenorientierte Ansätze in diesem Bereich Schwächen.

Tabelle 1: Abgleich von Informationsbedarfen mit existierenden Lösungsansätzen

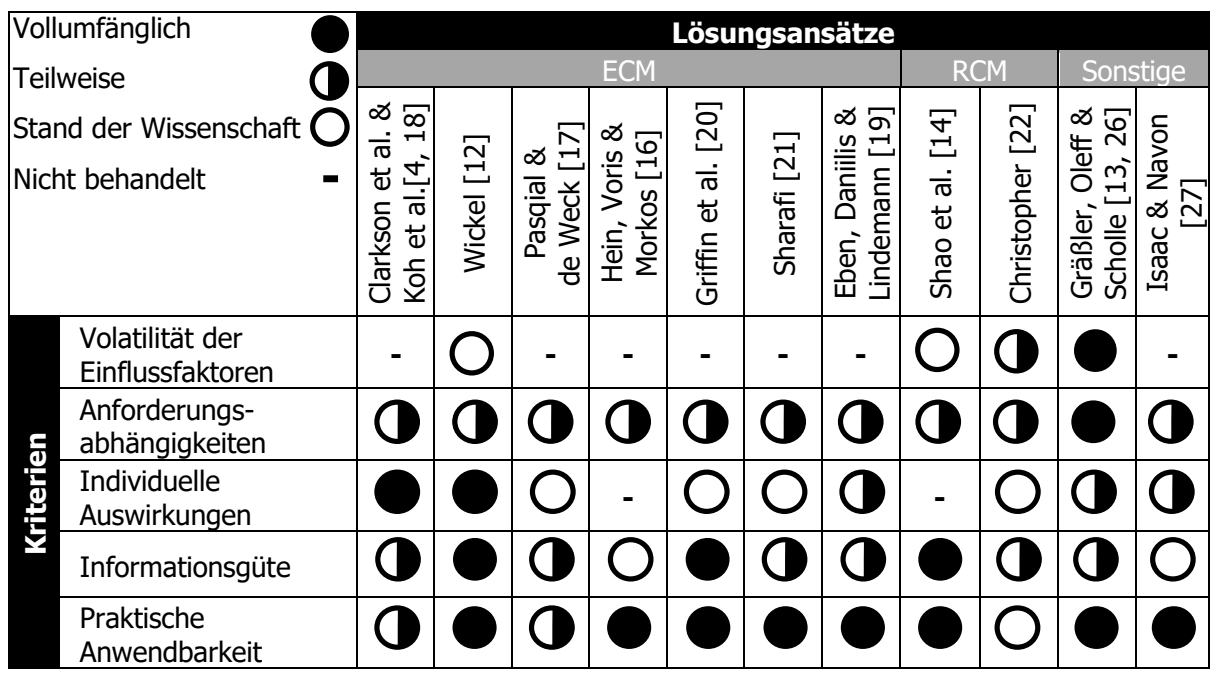

\section{Zusammenfassung und Ausblick}

In diesem Beitrag wurde ein Referenzmodell im Sinne der DRM für die Handhabung von Anforderungsänderungen vorgestellt, das die Informationsbedarfe einer risikoorientierten Anforderungsanalyse und -handhabung herleitet. Anhand des Referenzmodells können grundlegende Informationsbedarfe abgeleitet und die wesentlichen Einflussfaktoren identifiziert werden, deren detaillierte Ausarbeitung für interdisziplinäre Systeme steht aber noch aus. Die Informationsbedarfe sind die Volatilität der Einflussfaktoren, die Anforderungsabhängigkeiten und die individuellen Auswirkungen. Anschließend wurden die Bedarfe mit existierenden Ansätzen abgeglichen. Es zeigt sich, dass in einigen Bereichen Ansätze zur Informationserhebung vorhanden sind. Die Besonderheiten interdisziplinärer Systeme wie z.B. die Diversität der Datengrundlage 
aber noch unzureichend berücksichtigt sind. Die Forschungsfragen konnten somit beantwortet werden, dabei wird deutlich, dass existierende angepasst und neue Ansätze zur Befriedigung der Informationsbedarfe entwickelt werden müssen, die die Spezifika interdisziplinärer Systeme ausreichend berücksichtigen.

\section{Literatur}

[1] K. Pohl, Requirements engineering: Fundamentals, principles, and techniques. Heidelberg, New York: Springer, 2010.

[2] D. D. Walden, G. J. Roedler, K. Forsberg, R. D. Hamelin und Shortell, Thomas M., Systems engineering handbook: A guide for system life cycle processes and activities, $4^{\text {th }}$ ed.: Wiley, 2015.

[3] J. Fernandes, E. Henriques, A. Silva und Moss, Michael A., "Requirements change in complex technical systems: An empirical study of root causes," Res Eng Design, vol. 26, no. 1, pp. 37-55, 2015.

[4] P. J. Clarkson, C. Simons und Eckert, Claudia, "Predicting Change Propagation in Complex Design," J. Mech. Des., vol. 126, no. 5, p. 788, 2004.

[5] L. T. M. Blessing and Chakrabarti, Amaresh, DRM, a Design Research Methodology, $1^{\text {st }}$ ed. Guildford, Surrey: Springer London, 2009.

[6] K. Forsberg, H.M. Mooz und Cotterman, H., Visualizing Project Management: Models and Frameworks for Mastering Complex Systems, 2005.

[7] S. McGee and Des Greer, "Towards an understanding of the causes and effects of software requirements change: Two case studies," Requirements Engineering, vol. 17, no. 2, pp. 133-155, 2012.

[8] K. Pohl and Rupp, Chris, Basiswissen Requirements Engineering: Ausund Weiterbildung nach IREB-Standard zum Certified Professional for Requirements Engineering, 4 ${ }^{\text {th }}$ ed. Heidelberg: dpunkt, 2015.

[9] T. A. W. Jarratt, C. M. Eckert, N. H. M. Caldwell und Clarkson, P. J., "Engineering change: An overview and perspective on the literature," Res Eng Design, vol. 22, no. 2, pp. 103-124, 2011.

[10] B. Hamraz, N. H. M. Caldwell und Clarkson, P. John, "A Holistic Categorization Framework for Literature on Engineering Change Management," Syst. Engin., vol. 16, no. 4, pp. 473-505, 2013.

[11] S. Jayatilleke and Lai, Richard, "A systematic review of requirements change management," Information and Software Technology, vol. 93, pp. 163-185, 2018.

[12] M. C. Wickel, "Änderungen besser managen," Dissertation, 2017.

[13] I. Gräßler, C. Oleff und Scholle, Philipp, "Priorisierung von Anforderungen für die Entwicklung mechatronischer Systeme," in Fachtagung Mechatronik 2019, Paderborn, 2019, pp. 1-6. 
[14] F. Shao, R. Peng, H. Lai und Wang, Bangchao, "DRank: A semi-automated requirements prioritization method based on preferences and dependencies," Journal of Systems and Software, vol. 126, 2017.

[15] B. Morkos, P. Shankar und Summers, Joshua D., "Predicting requirement change propagation, using higher order design structure matrices: An industry case study," Journal of Engineering Design, vol. 23, no. 12, 2012.

[16] H. Hein, N. Voris und Morkos, Beshoy, "Predicting requirement change propagation through investigation of physical and functional domains," (eng), Research in Engineering Design, vol. 29, no. 2, 2018.

[17] M. C. Pasqual and Weck, Olivier L. de, "Multilayer network model for analysis and management of change propagation," Res Eng Design, vol. 23, no. 4, pp. 305-328, 2012.

[18] Y. Koh, M. Caldwell und Clarkson, John, "A method to assess the effects of engineering change propagation," (eng), Research in Engineering Design, vol. 23, no. 4, pp. 329-351, 2012.

[19] Eben, K. G. M., C. Daniilis und Lindemann, U., "Interrelating and Prioritising Requirements on Multiple Hierachy Levels," in Design 2010: $11^{\text {th }}$ International Design Conference, Dubrovnik - Croatia, May 17 - 20, 2010, D. Marjanović, Ed., Zagreb, 2010, pp. 1055-1064.

[20] M. Giffin et al., "Change Propagation Analysis in Complex Technical Systems," J. Mech. Des., vol. 131, no. 8, p. 81001, 2009.

[21] A. Sharafi, Knowledge Discovery in Databases: Eine Analyse des Änderungsmanagements in der Produktentwick/ung. Zugl.: München, Techn. Univ., Diss., 2012. Wiesbaden: Springer Gabler, 2013.

[22] D. F. X. Christopher, "Prediction of Software Requirements Stability Based on Complexity Point Measurement Using Multi-Criteria Fuzzy Approach," IJSEA, vol. 3, no. 6, pp. 101-115, 2012.

[23] M. Diederichs, Risikomanagement und Risikocontrolling, 3rd ed. München: Verlag Franz Vahlen, 2012.

[24] I. Gräßler, P. Scholle, J. Hentze und Oleff, Christian, "Semi-Automatized Assessment of Requirement Interrelations," in Proceedings of the DESIGN $201815^{\text {th }}$ International Design Conference, Dubrovnik, 2018, pp. 325-334.

[25] A. Albers et al., "Managing Systems of Objectives in the agile Development of Mechatronic Systems by ASD - Agile Systems Design," NordDesign, 2018.

[26] I. Gräßler, C. Oleff und Scholle, Philipp, "Methode zur Bewertung von Anforderungsänderungen additiv gefertigter Produkte," in Design for $X$ : Beiträge zum 29. DfX-Symposium, Tutzing, 2018, pp. 333-344.

[27] S. Isaac and Navon, Ronie, "Feasibility Study of an Automated Tool for Identifying the Implications of Changes in Construction Projects," J. Constr. Eng. Manage., vol. 134, no. 2, pp. 139-145, 2008. 